\title{
ON THE EFFECT OF RELATIVE DENSITY ON THE CRUSHING AND ENERGY ABSORPTION OF OPEN-CELL FOAMS UNDER IMPACT
}

1 Corresponding author

E-mail:skk@mail.utexas.edu 


\section{Abstract}

42 Micromechanically accurate random foamsare used to examine the effect of relative densityon

43 the crushing response and energy absorption of aluminum open-cell foams for a range of loading

44 rates. Random soap froth microstructures generated with the Surface Evolver software are

45 dressed with shear deformable beam elements with material distribution that mimics measured

46 values. Foam models of relative densities between $3.67 \%$ and $10.0 \%$ are crushed first quasi-

47 statically and then dynamically in a direct impact setting covering impact speeds that ranged

48 from sub- to super-critical.Under quasi-static crushing, the limit and plateau stresses and the

49 energy absorbed were found to to increase linearly with relative density while the densification

50 strains exhibits a modest linear decrease. In the shock regime, for all densities the proximal

51 stresses rise significantly with relative density whereas the distal ones follow the more modest

52 linear increase of the quasi-static limit stress. The Hugoniot strain depends strongly on impact

53 speed approaching asymptotically values corresponding to the densification of the material at the

54 high end. The energy induced to the material as it crosses the shock also increases also

55 parabolically with impact speed. It was further confirmed that given the shock front velocity-

56 impact speed Hugoniot the rest of the dynamic variables for each foam density can be estimated

57 from the fundamental jump conditions for shocks in solids. For all densities examined it was

58 illustrated that the transition from quasi-static to shock behavior is a process that spans several

59 impact speed. The transitional range moves to moderately higher velocities as the foam density 60 increases. 


\section{INTRODUCTION}

Cellular materials in general and foams in particularhave unique mechanical, thermal, acoustical and other properties derived from their cellular microstructures (e.g., see Gibson and Ashby, 1997). Mechanically they have compressive responses with a relatively low initial maximum stressfollowed by a stress plateau that extends to high strains. This behavior, in addition to their low density, is responsible for their wide use as cores in sandwich structures and in impact mitigation and blast protection applications (e.g., Ashby et al., 2000). It is now well established that the stress plateau, common to all but brittle cellular materials, is caused by localized crushing of cells that gradually spreads until the whole domain is crushed. In other words, the deformation is inhomogeneous and as a result does not represent a stress-strain response of the material (Klintworth and Stronge, 1988; Papka and Kyriakides, 1994, 1998; Gong et al., 2005,Jang and Kyriakides, 2009a). The stress plateau is followed by a progressively stiffening response (densification) during which additional crushing takes place but now in a more homogeneous manner.

Impact mitigation and blast protection naturally require that attention be also paid to the effect of inertia on the material response and its energy absorption characteristics. In the shock physics community it has long been established that material systems with adiabatic compression modulus that increases with further compression, such as that of cellular materials in the densification regime, can be expected to develop stable shocks (e.g., see Bethe, 1942; Herrmann, 1969; Knowles, 2002). Furthermore, the shock behavior can be fully represented by the Hugoniot of the material. Shock formation, accompanied by significant increase in the dynamic energy absorption capacity in cellular materials was first reported in the mechanics community by Reid and Peng [1997] from Taylor impact experiments on wood. Dynamic crushing on polymeric foams (e.g., Skews et al. 1991; Zaretsky and Ben-Dor, 1995; Zaretskyet al., 2012) and more recent impact experiments on open and closed cell metal foams (e.g., Tan et al., 2005a,b; Elnasriet al., 2007; Tan et al., 2012) reported shock formation above a certain critical impact speed (for a more complete review of the experimental literature see Barnes et al., 2014).

Until recently the modeling of the dynamic/shock behavior of cellular materials was

92 which the deformation in the densification regime is precluded ( $r$ - $p$-p-l:rigid-perfectly-plastic93 locking model,seeTan et al., 2005b) or, in more representative models, varies in a nonlinear 
94 manner with impact velocity (e.g.,Pattofattoet al., 2007; Karagiozovaet al., 2012; Karagiozova 95 and Alves, 2014). The adoption of the foam's quasi-static response as a stable material response 96 is clearly refuted by results from crushing experiments on metal foams from many sources 97 including Jang and Kyriakides [2009a] and Barnes et al. [2014] (see also earlier work on 98 honeycombs such as:Klintworth and Stronge, 1988;Papka and Kyriakides, 1994, 1998a, 99 1998b).Barnes et al. [2014] used high-speed photography to monitor the evolution of 100 deformation and propagation of shocks in direct and stationary impact experiments on Al-alloy 101 open-cell foams with a relative density of about $8 \%$. Shocks developed at impact speeds higher 102 than about $50 \mathrm{~m} / \mathrm{s}$. Direct measurements of shock velocity and strain behind the shock at 103 different impact speeds enabled the establishment of the Hugoniot, which in turn allows 104 calculation of all problem variables without resorting to a constitutive model. The Hugoniot 105 strain was shown to increase significantly with impact speed and the stress behind the shock to 106 increase quadratically. At impact speeds below the critical threshold localized crushing initiates 107 progressively at multiple sites, similar to quasi-static crushing, while the stress traces the usual 108 plateau. In summary, use of the quasi-static material response in modeling the shock behavior is 109 inappropriate.

110 The modeling of the quasi-static compressive response and crushing of random foams, 111 has evolved from models based on idealized microstructures such as variations of the periodic 112 Kelvin cell (Gong and Kyriakides, 2005; Jang et al. 2008; Jang and Kyriakides, 2009b) to more 113 representative random ones generated using the Surface Evolver software. These 114 referencesshowed that suitably calibrated Kelvin cell models can capture essentially all 115 properties from the initial elastic behavior, the stress at the onset of localization, the stress 116 plateau that follows and its extent, to densification. However, the crushing patterns are 117 influenced by the periodicity of the microstructure and differ from ones observed in experiments 118 on random foams. Gaitanaroset al. [2012] showed thatmicromechanically accurate random foam 119 models reproduce all aspects of the quasi-static crushing behavior of such materials including the 120 correct crushing behavior.

121 This type of random foam model was subsequently used in Gaitanaros and Kyriakides 122 [2014] to study the crushing behavior of the foam used in the experiments of Barnes et al. [2014] 123 under impact. The behavior observed in the experiments in the subcritical and supercritical 124 regimes was reproduced. This included the generation of the Hugoniot, the parabolic increase of 
125 the stress behind the shocks with impact speed, and the corresponding increase of the Hugoniot 126 strain. The model was subsequently used to conduct a wider parametric study that included a 127 detailed probing of the transition from quasi-static to shock type crushing. It is worth mentioning 128 that the work of the authors and co-workers on the crushing of space filling foams by impact was 129 preceded by micromechanical modeling of in-plane dynamic crushing of honeycombwhere 130 subcritical and shock behavior was observed. Specifically the works of Hönig and Stronge, 131 [2002a,b], Ruanet al. [2003] and Zouet al. [2009] connect well with the present studies.

132 It is widely understood that most mechanical and other properties of cellular materials 133 scale with their relative density (Gibson and Ashby, 1997). Thus the present study examines the 134 effect of the foam relative density on crushing and energy absorption at different loading rates 135 ranging from quasi-static to sub- and super-critical impact velocities. This is a natural extension 136 of our preceding work [2014] and uses the same type of discrete random foam model to develop 137 results in a range of densities of practical interest. The base material remains the same, Al-6101138 T6, and the open cell foam modelsare again generated via the Surface Evolver (Brakke, 1992).

139 The material distribution in the ligaments is assumed to follow the trends of the measurements 140 performed in Jang et al. [2008]with the basic dimensions being varied to design models with 141 relative densities ranging from $3.67 \%$ to $10.0 \%$ (range that is commercially available,see ERG 142 website).

\section{2. MODELING OF RANDOM FOAMS}

145 The open cell foams used in our studies are Al-6101-T6 ERG Duocel®) (see ERG

146 website). This product is cast using molds generated from nearly monodisperse polymeric foams. 147 Consequently, they have the same distribution of random polyhedra and polygonal sides as 148 polymeric and more generally liquid foams. This characteristic enabled us to generate models 149 with similar random microstructures from soap froths generated by the Surface Evolver (e.g., see 150 Kraynik et al., 2003, 2004). The skeletal version of the froth (e.g., see Fig. 1a) is "dressed" with 151 appropriate distributions of solid to match those of ligaments in foams analyzed. In particular we 152 start with a random soap forth with $12^{3}$ cells. The vertices of the cellsare joined with straight 153 lines and anisotropy of $\lambda=1.1$ is introduced by applying an affine deformation to the model in 154 the $x_{1}$-direction (see previous works of the group). The periodicity of the models is removed and 
155 the ligaments in the top and bottom surfaces are cropped so that their ends become co-planar 156 ending up with a domain size of $12 \times 10 \times 10$ cells (see Gaitanaroset al., 2012 for more details).

157

\subsection{Material Distribution in Ligaments and Discretization}

The straight ligaments are "dressed" with shear-deformable beams with circular cross sections and variable area along their length that follows the expression developed from measurements on a nearly $8 \%$ relative density foam reported in Jang et al. [2008], repeated here for completeness,

$$
A(\xi)=A_{O} f(\xi)=A_{o}\left(36 \xi^{4}+\xi^{2}+1\right), \quad \xi=x / \ell,
$$

where $A_{O}$ depends on the ligament length $\ell$ as follows:

$$
A_{o}=\bar{A}_{o} g(\eta)=\bar{A}_{o}\left(0.6633+0.264 \eta^{-2.5963}\right), \quad \eta=\ell / \bar{\ell}
$$

and $\bar{A}_{O}$ and $\bar{\ell}$ and the average values measured.

Modeling ligaments as beams leads to an overlap of material at the nodes. This is accounted for using the following expression for the relative density (Jang et al., 2008):

$$
\frac{\rho^{*}}{\rho}=k\left(\frac{R_{O}}{\ell}\right)^{n},
$$

where $R_{O}$ is the mid-span radius of the ligament. The parameters $k$ and $n$ depend on the anisotropy and for $\lambda=1.1$ take the values of 1.9543 and 1.7131 respectively. As mentioned above, the empirical expressions (1) and (2) came from measurements of ligament geometries in ERG Duocel ${ }^{\circledR}$ foams of relative density of about $8 \%$. We generate models with relative densities of $3.67 \%, 5.56 \%, 7.75 \%$ and $10.0 \%$ by assuming that this distribution of material remains the same. As in our previous works, the ligaments are placed in two length groups: shorter and longer than $\bar{\ell}$. The average lengths of each group are designated as $\bar{\ell}_{1}$ and $\bar{\ell}_{2}$ and the corresponding values of $A_{O}$ in (2) as $\left.A_{o}\right|_{\ell_{a}}, \quad \alpha=1,2$.

Theligaments are discretized with the Hughes-Liu [1981] linear beam element that is derived from the isoparametric 8-node solid element. Ligaments with $\ell<\bar{\ell}$ are discretizedusing 7 elements and those with $\ell>\bar{\ell}$ with 9 elements as in Gaitanaros et al. [2012]. All elements have a uniform circular cross sectional area based on the following: 
183 with $\left.f(\xi)\right|_{\ell_{\alpha}}, \quad \alpha=1,2$ taking the same values listed in Table 1 of the reference given above 184 (e.g., see model foam slice in Fig. 1b).

185 Proper modeling of contact between crushing ligaments is essential in order to reproduce correctly the shock front, its propagation and the compaction of the material behind it.This is

187 achieved through the beam-to-beam contact algorithm ofLS-DYNA adopted along with a penalty 188 formulation with an interface stiffness chosen for numerical stability to be one order of 189 magnitude less than the stiffness of the contacting elements. Coulomb friction with a coefficient 190 of 0.4 was found to yield good numerical stability of the solutions and is used in all calculations.

191 As noted above, the use of beam elements leads to excess material at the nodes. This 192 excess material was corrected for the calculation of the density of the model foam but the 193 overlaps remain in theFE model. These overlaps at the nodes cause initial 194 interpenetrationsbetween beam elements and non-physical contacts that lead to numerical 195 instabilities of the contact algorithm. This difficulty was bypassed by excluding the overlapping 196 elements with the scaled assigned density from developing contact. Since for higher densities 197 beams are thicker and the overlap is more significant, density is scaled in four elements per 198 ligament for the $7.75 \%$ and $10.0 \%$ foams but in two elements for the $3.67 \%$ and $5.56 \%$ foams.

As in our previous works the Al-6061-T6 base material is modeled as a finitely 200 deforming elastic-plastic solid calibrated to the true-stress-logarithmic strain version of the 201 engineering stress-strain response with the following Ramberg-Osgood parameters: $E=10^{4} \mathrm{ksi}-$ $20269 \mathrm{GPa}, \sigma_{y}=28 \mathrm{ksi}-193 \mathrm{MPa}, n=48$ (properties measured in a tensile test on a specimen cast 203 together with the foam in Jang and Kyriakides, 2009b).

\section{RESULTS}

\section{$206 \quad 3.1$ Quasi-Static Crushing}

207 The models for the four foam densities are first crushed quasi-statically between two 208 rigid surfaces in the manner described in Gaitanaros and Kyriakides [2012]. The calculated 209 nominal stress-shortening responses $\left(\sigma-\delta / h_{O} ; h_{O}\right.$ is the undeformed height of the model $)$ are

210 plotted in Fig. 2. ( $\sigma$ is the net force acting on the rigid contacting plane divided by the initial 211 cross sectional area of the model). The four responses are qualitatively similar, all displaying a 
212 stiffand stable elastic branch, a local pressure maximum followed by relatively smooth stress 213 plateau and a densification regime. As expected, the whole response moves to a higher stress 214 level as the density increases and the average strain at densification decreases (marked with "“"). 215 Figure 3 shows the undeformed and three deformed configurations for each of the four 216 foammodels. They are cross sectionsabout two cells thick taken approximately from the center of 217 each domain.It is readily noticed that as density increasesligaments become less slender. Images 218 I correspond to $\delta / h_{O}=10.0 \%$ and are marked in Fig. 2 with " $\bullet$ ". Since the models originated 219 from the same random soap froth, localized cell crushing initiated at the same location in each 220 model. Subsequently,crushing propagates upwards while cell collapse has commenced in a 221 second band above the first one that can be seen in images II at $\delta / h_{o}=30 \%$ (marked with "凶" in 222 Fig. 2). In summary then the behavior observed thus far is very similar to that reported in 223 Gaitanaros and Kyriakides [2012] for foams with densities of about 8\%. Images III 224 approximately correspond to the point when cell crushing has fully consumed each model. It 225 coincides with the upswing in the stress of each response in Fig. 2 and we designate it as the 226 onset of densification and the corresponding value of $\delta / h_{o}$ as $\varepsilon_{D}$ (marked with "m" in Fig. 2).

227 As the density increases, $\varepsilon_{D}$ decreases which is also evident from the height of the densified 228 configurations III in Fig. 3. The height of the model with relative density of $3.67 \%$ is $0.37 h_{o}$ 229 while that of the one with $10.0 \%$ is $0.52 h_{o}$. Clearly, as the density increases, the ligaments 230 become thicker and during crushing come into contact earlier which translates into a shorter 231 stress plateau and thus a smaller densification strain.

232 Table 1 Quasi-static crushing characteristics of Al-alloy foams of several densities.

\begin{tabular}{|c|c|c|c|c|}
\hline$\frac{\rho^{*}}{\rho}(\%)$ & $\begin{array}{c}\sigma_{I} \mathrm{psi} \\
(\mathrm{MPa})\end{array}$ & $\begin{array}{c}\sigma_{P} \mathrm{psi} \\
(\mathrm{MPa})\end{array}$ & $\varepsilon_{D}$ & $\begin{array}{c}U_{O} \mathrm{psi} \\
(\mathrm{MPa})\end{array}$ \\
\hline \hline 3.67 & $\begin{array}{c}105 \\
(0.72)\end{array}$ & $\begin{array}{c}103 \\
(0.71)\end{array}$ & 0.63 & $\begin{array}{c}65.1 \\
(0.45)\end{array}$ \\
\hline 5.56 & $\begin{array}{c}259 \\
(1.76)\end{array}$ & $\begin{array}{c}255 \\
(1.76)\end{array}$ & 0.58 & $\begin{array}{c}152.1 \\
(1.05)\end{array}$ \\
\hline 7.75 & $\begin{array}{c}397 \\
(2.74)\end{array}$ & $\begin{array}{c}398 \\
(2.74)\end{array}$ & 0.54 & $\begin{array}{c}220.8 \\
(1.52)\end{array}$ \\
\hline 10.04 & $\begin{array}{c}586 \\
(4.04)\end{array}$ & $\begin{array}{c}589 \\
(4.06)\end{array}$ & 0.48 & $\begin{array}{c}291.4 \\
(2.01)\end{array}$ \\
\hline
\end{tabular}


The initial stress maximum represents the onset of instability in compressed foams that 235 we have designated as the initiation stress, $\sigma_{I}$. In dynamic crushing it represents the stress 236 barrier that must be exceeded by an incoming stress wave for a shock to develop. The stress 237 plateau, designated as $\sigma_{P}$, that follows and its extent, in other words $\varepsilon_{D}$, represent the energy 238 absorption capacity of the foam. The energy absorption capacity per unit volume, designated as $239 U_{O}$, is established by integrating the response from 0 to $\varepsilon_{D}$. The calculated values of the four 240 variables are listed in Table 1 while their trends can also be seen in Fig. 4a and 4b where they are 241 plotted against relative density. Thus, $\sigma_{I}$ and $\sigma_{P}$, each normalized by the base material yield 242 parameter $\sigma_{y}$ follow a linear trajectory that does not pass through the origin presumably 243 because for very low densities the instability is governed by elastic material behavior. With the 244 present definition of the densification strain their values are similar (Jang and Kyriakides, 2009a 245 used an alternate definition of $\varepsilon_{D}$ that accentuates the differences between the two stress 246 variables). Their absolute values as well as the linear dependence on $\rho^{*} / \rho$ are in general 247 agreement with measured values reported in Jang and Kyriakides [2009a] and calculated values 248 of $\sigma_{I}$ in Jang et al. [2010]. Gibson and Ashby [1997] report two powerlaw expressions for $249 \sigma_{P} / \sigma_{y}$ based on scaling arguments that are widely used in the design of foams and sandwich 250 structures (e.g., Eq. (5.27a) $\sigma_{P} / \sigma_{y} \approx 0.3\left(\rho^{*} / \rho\right)^{3 / 2}$; no distinction is made between $\sigma_{I}$ and $\sigma_{P}$

251 ). They were found to disagree with the present results as well as with the experimental results of 252 Jang and Kyriakides [2009a]both qualitatively and quantitatively, underpredicting them by 253 factors upwards of 2. The densification strain plotted in Fig. 4a is seen to decrease essentially 254 linearly with relative density. Gibson and Ashby's expression for $\varepsilon_{D}$ (Eq. (5.30): $\left.255 \varepsilon_{D}=1-1.4\left(\rho^{*} / \rho\right)\right)$ also decreases linearly with relative density, however it over-predicts the 256 present results by more than $30 \%$. Driven primarily by the increase in the plateau stress, the 257 energy absorption in Fig. 4b increases linearly with relative density. Thus for example, $U_{O}$ for 258 the $10.0 \%$ density foam is nearly 4.5 times larger than that of the $3.67 \%$ density foam. 259

\subsection{Shock Formation Under Impact}


The same four models are now crushed dynamically in a direct impact setting (see Fig.

262 1b). In each casethe specimen is assigned a constant velocity towards a stationary rigid surface.

263 The objective is to understand the effect of shocks on the induced stresses and deformations in

264 foams of different densities as a function of impact speed. The tools that will be used to analyze

265 the results are the classical jump conditions in Lagrangian form representing conservation of 266 mass, momentum, and energy applied to plane longitudinal shocks summarized below (Davison, 267 2008):

$$
\rho_{O} \dot{S}\left[\left[\frac{1}{\rho}\right]\right]+[[V]]=0,
$$

$$
\rho_{O} \dot{S}[[V]]+[[\sigma]]=0
$$$$
\rho_{O} \dot{S}\left[\left[U+\frac{1}{2} V^{2}\right]\right]+[[\sigma V]]=0
$$

271 where $[[g]]=g^{+}-g^{-}$is the jump operator with $g^{+}$and $g^{-}$representing the value of a variable 272 ahead and behind a discontinuity; $S$ is the position of the discontinuity in the 273 undeformedconfiguration, and $\dot{S}$ its velocity. The variables $\{\rho, V, \sigma, U\}$ are respectively the 274 mass density, particle velocity, nominal stress, and strain energy density.

275 Figure 5 shows an image of such a foam model that has been partially crushed by a 276 shock. The rigid plane on the top is moving together with the uncrushed foam at a velocity $V^{+}$ 277 while the one on the bottom is fixed in space.As the foam is crushed, cells located at the front 278 experience a fast deceleration from $V^{+}$to almost zero velocity. The horizontal dashed red line 279 shows the best estimate of the position of the shock, in this particular locationin the width of the 280 model. Similar estimates of the position of the shock are conducted at different locations across 281 the width and from the corresponding skeletal views. The average of these estimates is $h_{C}(t)$ and 282 then $h_{i}(t)=h_{b}(t)-h_{c}(t)$. The position of the front in the undeformed configuration is then $283 s(t)=h_{O}-h_{i}(t)$, and its velocity is the time derivative $\dot{s}(t)$. As in Barnes et al. [2014], assuming 284 that the transverse strain is negligibly small

$$
\left(\frac{1}{\rho^{+}}-\frac{1}{\rho^{-}}\right)=\left(\frac{1}{\rho_{O}}-\frac{h_{C}}{s \rho_{O}}\right)=\frac{\varepsilon_{H}}{\rho_{O}}
$$


where $\rho_{O}$ is the initial density of the material assumed to remain unchanged ahead of the shock and $\varepsilon_{H}$ is the strain behind the shock, named the Hugoniot strain. Using (6), each of Eqs. (5) can be respectively expressed as follows:

$$
\dot{s} \varepsilon_{H}=\left(V^{+}-V^{-}\right)
$$

$$
\sigma^{+}=\sigma^{-}-\rho_{O} \dot{s}\left(V^{+}-V^{-}\right)=\sigma^{-}-\rho_{O} \frac{\left(V^{+}-V^{-}\right)^{2}}{\varepsilon_{H}},
$$

$$
\rho_{O}\left(U^{+}-U^{-}\right)=\frac{1}{2}\left(\sigma^{+}+\sigma^{-}\right) \varepsilon_{H}
$$

In the first set of simulations, an impact velocity of $90 \mathrm{~m} / \mathrm{s}$ is chosen to ensure shock

294 proximal $\left(\sigma^{-}\right)$and distal $\left(\sigma^{+}\right)$ends respectively for the four foams. Qualitatively, the characteristics of the two pairs of responses are similar to those reported in Gaitanaros and Kyriakides [2014] in their simulations of specific experiments. Briefly, each of the stresses behind the shock exhibits an initial spike with a quick decent down to a stress plateau with several undulations. The events associated with the four responses are summarized in Fig. 7 that shows the undeformed and three deformed slices of each model that correspond to the bullets marked on the responses, labeled I, II and III (marked in Fig. 6a with $\bullet, \mathbf{\Delta}, \mathbf{\square})$. Images I show the early formation of the shock; images II show the shock having crushed a significant part of the specimen; and set III shows the shock having just reached the end of the specimen when the $\sigma^{-}$ stressesin Fig. 6a start rising. The $\sigma^{-}$stress plateaus increase with density going from approximately $200 \mathrm{psi}(1.38 \mathrm{MPa})$ for the lowest density to approximately $950 \mathrm{psi}(6.55 \mathrm{MPa})$ for the $10.0 \%$ relative density. Because the soap froth from which the four models originated is the same, the stress undulations occur at the same times (partly associated with the nearly planar shock crossing a relatively course microstructure). However, as is evident from the height of images III, the shock induced strain decreases as the density increases and consequently the corresponding plateaus terminate earlier.

The stress history recorded at the distal end, $\sigma^{+}$, that represents the stress in the essentially undeformed (only elastic deformation) part of the specimen behind the shock is

313 is somewhat delayed from the time of first impact due to the time required for the stress wave to 
314 traverse the length of the model. They trace relatively flat plateaus that again increase with 315 density but the levels are significantly lower than those recorded at the proximal end in Fig. 6a.

316 The difference can be explained using Eq. (7b) above. The plateaus again finish with a sharp rise

317 in the stress close to the time that the shock has traversed the model. This termination of the

318 plateaus occur somewhat earlier than the ones at the proximal end, possibly because as the shock 319 approaches the rigid plane it interacts with it. Drawn with a dashed line in Fig. 6bis the initiation 320 stress $\sigma_{I}$ corresponding to each density (Table 1 ). Each plateau is seen to be bounded by $\sigma_{I}$, 321 which is in agreement with the conclusion drawn in Gaitanaros and Kyriakides [2014] for a 322 relative density of about $8 \%$ (similar observation made from modeling of impact of regular 323 honeycomb in Zou et al., 2009).

\subsection{Effect of Relative Density on the Hugoniot}

The random models are now used to generate first the $V^{+}-\dot{s}$ Hugoniot for the four relative densities. This is achieved by performing a series of constant velocity direct impact

328 simulations for each density in the velocity range of $30<V^{+} \leq 160 \mathrm{~m} / \mathrm{s}$ (the lower velocity varies

329 with density in order to ensure that shocks develop). The numerical procedure used is the same 330 as that outlined in Section 3.2. The values of $\dot{s}$ calculated for different impact speeds for the four 331 foam densities are are listed in Table 2. They are also plotted against each other in Fig.8 for each 332 density. All four sets of results form a nearly linear locus, a result that is in agreement with the 333 trend of the experimental valuesfrom the nearly $8 \%$ relative density $\mathrm{Al}$ foam in Barnes et al. 334 [2014]. Included with dashed lines are linear fits of the numerical results that can be represented 335 by

$$
\dot{s}=A+B V^{+}
$$

337 The fit parameters are listed in Table 3 along with the $R^{2}$ values of the fits. Again, the nearly 338 perfect fits support the linearity of these Hugoniot curves. The four fits have nearly equal slopes 339 but different intercepts. Thus, as the density increases the shock velocity for a given impact 340 speed increases. For denser materials, the intercept is usually thought to be the wave speed of the 341 solid (e.g., Davison, 2008). In foams with low densities like the ones impacted in this study, this 342 limit is not recovered. Our best explanation at the present is that this is because the material 343 deforms inhomogeneously at impact speeds lower than the critical value for shock formation 
344 (Barnes et al., 2014). Hugoniot curves(8) are characteristic of each foam and constitute the 345 minimum amount of information required to evaluate the other shock propagation variables in 346 such problems.

Table 3 Hugoniot linear fit parameters

\begin{tabular}{|c|c|c|c|}
\hline$\frac{\rho^{*}}{\rho}(\%)$ & $\begin{array}{c}A \\
\mathrm{~m} / \mathrm{s}\end{array}$ & $B$ & $R^{2}$ \\
\hline \hline 3.67 & 18.76 & 0.960 & 0.9994 \\
\hline 5.56 & 26.03 & 0.974 & 0.9997 \\
\hline 7.75 & 29.62 & 0.987 & 0.9996 \\
\hline 10.04 & 36.61 & 0.9648 & 0.9998 \\
\hline
\end{tabular}

349

350

351

352

353

The results of these simulations are also used to generate other representations of the Hugoniot. Thus Fig. 9 shows a plot of the strain behind the shock vs. the impact speed that we call the $V^{+}-\varepsilon_{H}$ Hugoniot. The strains behind the shocks at different impact speeds can be obtained directly from the simulations of each foam density in the manner described in Section 3.2 and the associated Fig. 5. The measured values are plotted with solid bullets in the figure and are also listed in Table 2. They exhibit the same strong increase with impact speed observed in the experiments of Barnes et al. [2014] (see also similar trend in Fig. 7 of Zheng et al., 2014from dynamic crushing simulations of an idealized closed cell model). At higher speeds each set of results appears to asymptotically approach full densification of the material. However, we refrain from extending the numerical study to higher velocities because the lateral strain tends to increase while simultaneously our contact algorithm and strategy begin to lead to some selfpenetration at the nodes.

Marked on the ordinate with empty symbols are the strains, $\varepsilon_{D}$, at the completion of the inhomogeneous crushing phase of each foam under quasi-static loading. It is interesting that the shock-induced strains tend to approach the corresponding value of $\varepsilon_{D}$ from above, which can thus be viewed as a lower bound for $\varepsilon_{H}$.

In order to test once more the characteristic nature of the $V^{+}-\dot{s}$ Hugoniot, the linear fit (8) along with the constants in Table 3are used in Eq. (7a) to evaluate the Hugoniot strain from the following: 


$$
\varepsilon_{H}=\frac{V^{+}}{A+B V^{+}} .
$$

370 The results are plotted in Fig. 9with solid lines together with each set of the independently measured values. Expressions (9) are seen to track the trend of the data quite well for all four 372 densities.

373 The stresses behind and ahead of the shock are important in the design of impact 374 mitigation applications and are considered next. Accordingly Fig. 10 shows the $V^{+}-\sigma^{ \pm}$ 375 Hugoniot, generated using the $\sigma^{-}$and $\sigma^{+}$stresses measured directly from the numerical 376 simulations (force acting on respective plane divided by the undeformed cross sectional area of 377 the model foam). The $\sigma^{-}$stresses are represented by solid symbols and the $\sigma^{+}$with the 378 corresponding open ones. The stresses behind the shock exhibit a quadratic increase with 379 velocity for all densities. These trends are in agreement with the experimental results of Barnes 380 et al. [2014] and similar results in Tan et al. [2012] both for the same Duocel ${ }^{\circledR}$ Al-alloy foams 381 that the microstructure of the present models is based on. The trajectories move to higher stress 382 levels as the density increases and the increase with impact speed becomes stronger.

383 The stresses behind the shock remain nearly constant for all densities again in concert 384 with experimental observations in Barnes et al. [2014]. Included in the figure on the ordinate are 385 theinitiations stresses from the quasi-static crushing calculation of each foam model ( $\sigma_{I}$, in 386 Table 1). Each is seen to correspond to the level of the corresponding $\sigma^{+}$, which supports the 387 premise that $\sigma_{I}$ can be viewed as an upper bound for $\sigma^{+}$.

388 It is also worth examining the veracity of the basic Hugoniot in (8) in estimatingthe $389 V^{+}-\sigma^{ \pm}$Hugoniot. This can be achieved by substituting (8) in to (7b) leading to

$$
\sigma^{-}=\sigma^{+}+\rho_{O} V^{+}\left(A+B V^{+}\right)
$$

392 In Barnes et al. [2014] we recommended that in the absence of measured values of $\sigma^{+}$they be 393 replaced by $\sigma_{I}$ in (10). This substitution produced trajectories that slightly overpredict the 394 measured stresses in Fig. 10. This is explained by observing that the calculated $\sigma^{+}$histories for $39590 \mathrm{~m} / \mathrm{s}$ impact speed in Fig. 6 match $\sigma_{I}$ for the early part of the impact period but drop 
somewhat in the latter part. The $\sigma^{+}$values in Table 2 and in Fig. 10 represent the mean value of

397 these plateaus and as a result they are somewhat lower than $\sigma_{I}$. Furthermore, the difference

398 decreases as the impact speed increases. For these reasons in the present predictions of $\sigma^{-}$ 399 through (10) we adopt the average value of the four $\sigma^{+}$values measured for each density. The 400 calculated expressions are drawn in solid lines in Fig. 10. They are seen to track quite adequately 401 the measured values, slightly overpredicting the ones corresponding to the highest density foam.

The energy expended across the shock can be evaluated using measured values of stresses and strains in $(7 \mathrm{c})$ as follows:

$$
U_{o}=\rho_{O} U^{-}=\frac{1}{2}\left(\sigma^{+}+\sigma^{-}\right) \varepsilon_{H}
$$

405 This expression is nicely illustrated by the Rayleigh line construction in Fig. 11 that shows 406 stresses $\sigma^{+}$and $\sigma^{-}$plotted against $\varepsilon_{H}$. The four Rayleigh lines are for $90 \mathrm{~m} / \mathrm{s}$ impacts. The 407 calculated values for the four foam densities are listed in Table 2 and are used in Fig. 12 to 408 generate the energy per unit volume-velocity Hugoniot, $V^{+}-U_{O}$. The energy density, $U_{O}$, is 409 seen to increase significantly with impact speed for all foam densities analyzed. This is a trend 410 that mimics that observed in the experimental results of Barneset al. [2014]. Using (11)and the $411 V^{+}-\dot{s}$ Hugoniotone obtains:

$$
U_{O}=\frac{\sigma^{+} V^{+}}{A+B V^{+}}+\frac{1}{2} \rho_{O} V^{+2}
$$

413 Predictions based on this expression are included with solids lines in Fig. 12 using again the 414 average of the calculated values of $\sigma^{+}$for each density(does slightly better than adopting $\sigma_{I}$ 415 instead).The predictions are seen to reproduce the trend of the measured values, overestimating 416 them slightly for the denser foam.

\subsection{Transition from Subcritical Velocities to Shock Formation}

In their study of an $8 \%$ relative density foam with similar microstructure to the present ones,Gaitanaros and Kyriakides [2014] demonstrated that at lower impact speeds the foam 421 crushes in a manner that is very similar to that observed under quasi-static loading. Under 422 impact, deformation localizes at the weakest site in the model. When ligaments come into 423 contact in the collapsed cells, crushing spreads to neighboring cells or initiates at another 
424 location. This continues until the whole domain is crushed while the stress traces a relatively flat 425 plateau like the ones in Fig. 2. Subsequently, the now densified material starts deforming 426 essentially homogenously at an increasing stress. As the impact speed increases, the first signs of 427 shock formation is that crushing initiates first at the proximal end while the stress recorded there 428 moves gradually to a higher level than the quasi-static crushing stress plateau. In this transitional 429 velocity regime, localization at an alternate site is also possible. The formation of a shock that 430 traverses the specimen consistently from one end to the other is delayed until a higher impact 431 speed is reached. In other words, the transition to shock formation is a process that spans several 432 impact speeds. These observations were confirmed by the experiments reported in Barnes et al. 433 [2014].

434 In the present study the four density foam models were used to further scrutinize these 435 observations and establish how foam density influences this transition. The results of this effort 436 confirm that the transition to shock formation is a process. Furthermore, since change in density 437 results in nearly parallel $V^{+}-\dot{s}$ Hugoniot lines with only a relatively modest increase in the 438 intercept, the transitional velocity range was accordingly found not to be altered significantly. 439 Thus, in order to highlight differences between foams of different densities, we will compare 440 here results from the highest and lowest densities analyzed. To this end stress-normalized 441 displacement responses for the $3.67 \%$ and $10.0 \%$ relative density foam impacted at different 442 speeds in the transitional regime are plotted in Figs. 13a and 13b respectively. The lower density 443 responses are for quasi-static (QS) crushing and at impact speeds of 15, 25 and 35m/s and the 444 higher density foams for QS, 25, 35 and $50 \mathrm{~m} / \mathrm{s}$. Accompanying each response is a deformed 445 configuration that appears inFig. 14 corresponding to $\delta / h_{O} \approx 15 \%$ and marked on the response 446 with a solid bullet.

447 In the case of the $3.67 \%$ relative density foam, impact at $15 \mathrm{~m} / \mathrm{s}$ causes an initial stress 448 spike but the rest of the response traces a plateau that is at the same level as that for quasi-static 449 crushing. Furthermore, the images in Fig. 14 show crushing to start localizing at the same 450 location where collapse initiated quasi-statically. For impact at $25 \mathrm{~m} / \mathrm{s}$ deformation localized at 451 the same location as the QS case but concurrently some partial collapse of the cells adjacent to 452 the impact plane takes place. This has the effect of lifting the response slightly. By the time that 453 corresponds to the image shown in Fig. 14, deformation has spread to the lower fifth of the 454 domain. In other words, inertial effects have started to influence the deformation patterns and to 
increase the stress at the proximal end. When the impact speed is increased to $35 \mathrm{~m} / \mathrm{s}$, the 456 proximal stress plateau recorded moves to a higher level (by about 10\%) implying a larger 457 influence of inertia. Indeed, the corresponding image in Fig. 14, shows collapse initiating at the 458 impacted plane, and subsequently to propagate as a shock in the manner shown in Fig. 7. We can 459 thus claim that for this density inertial effects start altering the behavior at $25 \mathrm{~m} / \mathrm{s}$ leading to shock formation at $35 \mathrm{~m} / \mathrm{s}$.

The $10 \%$ relative density foam exhibits the same general behavior but the transition shifts to a higher impact speed. Thus, at $25 \mathrm{~m} / \mathrm{s}$ the response is similar to the quasi-static one with crushing initiating at the same weak site in Fig. 14. At $35 \mathrm{~m} / \mathrm{s}$, the response moves to a slightly higher level and the same confused crushing patterns develop at the lower end of the domain as those seen for $25 \mathrm{~m} / \mathrm{s}$ for the lower density foam. Impact at $50 \mathrm{~m} / \mathrm{s}$ causes a clear shock to develop at the proximal end that propagates, crushing the whole domain. In other words, for the $10 \%$ relative density foam the transition from quasi-static to shock behavior has moved to between $35 \mathrm{~m} / \mathrm{s}$ and $50 \mathrm{~m} / \mathrm{s}$.

\section{SUMMARY AND CONCLUSIONS}

Gaitanaros and Kyriakides [2012] demonstrated that micromechanically accurate models of random foams can reproduce all aspects of their quasi-static crushing behavior from the onset of localization to homogeneous compaction during densification. The same type model was also shown [2014] to be capable of reproducing the behavior in experiments on foam under impact in both the subcritical and supercritical regimes. Thus in the shock forming regime the generation of the Hugoniot, the parabolic increase of the stress behind the shocks with impact speed, and the corresponding increase of the Hugoniot strain were reproduced with accuracy for the $8 \%$ relative density foam considered. Since most mechanical properties of cellular materials scale with relative density, the present study used similar discrete models to examine its effect on the crushing at different loading rates ranging from quasi-static to sub- and super-critical impact speeds.

Random soap froth models generated using the Surface Evolver software were "dressed"

$4847.75 \%$ and $10.0 \%$. The main modeling parameters derived from the microstructural measurements of Jang et al. [2008] were adopted in this process. In addition, a geometric 
anisotropy of $\lambda=1.1$ was assumed for all densities. Under quasi-static compression between

487 rigid planes, the four models exhibited the same general behavior consisting of: an initial elastic 488 regime that terminates into a local stress maximum that represents the onset of localized cell 489 collapse,followed by an extended stress plateau during which collapse propagates throughout the 490 domain. Once all cells have collapsed (densification) the stress risesonce more and deformation 491 is essentially uniform. For the density range considered the initiation, plateau stresses and energy 492 absorption capacity increase linearly with density while the densification strain decreases 493 linearly to some extent. The quantitative dependence of these variables to $\rho^{*} / \rho$ was found to 494 differ significantly fromscaling estimates in Gibson and Ashby [1997].

495 The same models were crushed dynamically in a direct impact setting where each model impacts a stationary rigid plane at a contrast velocity. When the impact speed is high enough, a 497 shock that consists of a narrow layer of crushed cells develops in the model foam. It initiates at 498 the impacted surface and propagates at a constant velocity towards the distal end until the whole 499 specimen is crushed. Calculations at impact speeds in the range of about $30-160 \mathrm{~m} / \mathrm{s}$ were 500 performed for each foam density. The shock velocity, the stress behind and ahead of the shock, 501 the strain induced by the shock and the energy expended across it were established as a function 502 of impact speed. This facilitated the generation of several representations of the Hugoniot for 503 each foam density that can be summarized as follows:

504 - The shock velocity-impact speed Hugoniot is linear for all densities with essentially the same 505 slope and an intercept that increases with density.

506 - The stress behind the shock grows in a quadratic manner with impact speed while the stress 507 ahead of the shock remains essentially constant at a value bounded by the initiation stress at 508 quasi-static crushing. The trend for the proximal stress increase significantly with density 509 while that at the distal end follows the linear growth of $\sigma_{I}$.

510 - The Hugoniot strain depends strongly on impact speed approaching asymptotically values 511 corresponding to the densification of the material. At the lower end the quasi-static 512 densification strain appears to bound the dynamic strain values. The trend is the same for 513 each density but moving to higher values as the density decreases.

514 - The crushing energy density also grows parabolically with impact speed. The trend applies to 515 all densities considered with the net energy absorbed increasing significantly with density. 
- The linear shock front velocity-impact speed Hugoniots were used in the fundamental jump conditions to estimate the rest of the dynamic variables for each foam density. The predictions follow the calculated results quite closely, as was the case in Barnes et al. [2014].

The transitional regimes of impact speeds between quasi-static and shock behavior were examined in some detail. It was confirmed that the transition to shock formation is a process that spans a range of impact speeds for all densities. At the lower end the crushing behavior and stress levels are like those in quasi-static crushing while at the higher end the proximal stress increases, crushing initiates at the impacted plane and propagates across the specimen as a shock. For the $3.67 \%$ relative density foam, this transition started at impact speeds of approximately 25 $\mathrm{m} / \mathrm{s}$ and finished at $35 \mathrm{~m} / \mathrm{s}$. For the $10 \%$ relative density foam it was shifted to $35-50 \mathrm{~m} / \mathrm{s}$.

\section{ACKNOWLEDGMENTS}

The work reported was supported in part by the National Science Foundation through Grant CMMI-1029575and by the University of Texas at Austin. This support is acknowledged with thanks. The authors would like to thank Andrew M. Kraynik for generating the soap froth models used in this study and for discussions and long term cooperation in mechanics of foams.

\section{REFERENCES}

Ashby, M.F., Evans, A., Fleck, N.A., Gibson, L.J., Hutchinson, J.W. and Wadley, H.N.G. (2000). Metal Foams: A Design Guide. Butterworth-Heinemann.

Brakke, K.A., 1992. The Surface Evolver. Experimental Mathematics1, 141-165. Available at http:/www.susqu.edu/facstaff/b/brakke/evolver/

Barnes, A.T., Ravi-Chandar, K., Kyriakides, S., Gaitanaros,S., 2014. Dynamic crushing of aluminum foams: Part I - Experiments. Int’l J. Solids \& Structures, 51:1631-1645.

Bethe, H., 1942. On the theory of shock waves for an arbitrary equation of state. Report No. 545 for the Office of Scientific Research and Development, Serial No. NDRC-B-237; reproduced in Classic Papers in Shock Compression Science, J.N. Johnson and R. Cheret (Eds), Springer, 1998, 421-492).

Davison, L. 2008. Fundamentals of Shock Wave Propagation in Solids. Springer.

Elnasri, I., Pattofatto, S., Zhao, H., Tsitsiris, H., Hild, F. and Girard, Y., 2007. Shock enhancement of cellular structures under impact loading: Part I experiments. J. Mechanics Phys. Solids 55, 2652-2671. 
ERG website: http://www.ergaerospace.com/Aluminum-properties.htm

Gaitanaros, S., Kyriakides, S., Kraynik, A.M. 2012. On the crushing response of random opencell foams. Int'l J. Solids Struct.49, 2733-2743.

Gaitanaros, S., Kyriakides, S., 2014. Dynamic crushing of aluminum foams: Part II - Analysis. Int'l J. Solids \& Struct., 51, 1646-1661.

Gibson, L.J., Ashby, M.F., 1997. Cellular Solids: Structure and Properties, 2nd Ed. Cambridge University Press.

Gong, L., Jang, W.-Y., Kyriakides S., 2005. Compressive response of open-cell foams. Part I: Morphology and elastic properties. Int'l J. Solids \& Structures 42, 1355-1379.

Gong, L., Kyriakides S., 2005. Compressive response of open-cell foams. Part II: Initiation and evolution of crushing. Int'l J. Solids \& Structures 42, 1381-1399.

Herrmann, W., 1969. Constitutive equations for the dynamic compaction of ductile porous materials. J. Appl. Physics 40, 2490-2499.

Hönig, A., Stronge, W.J. 2002a. In-plane dynamic crushing of honeycomb Part I: crush band initiation and wave trapping. Int'l J. Mech. Sciences44, 1665-1696.

Hönig, A., Stronge, W.J. 2002b. In-plane dynamic crushing of honeycomb Part II: application to impact. Int'l J. Mech. Sciences44, 1697-1714.

Hughes, T.J.R. and Liu, W.K., 1981. Nonlinear finite element analysis of shells-Part II. Twodimensional shells. Computer Meth. Applied Mech. Eng.27, 167-181.

Jang, W.-Y., Kraynik, A.M., Kyriakides, S., 2008. On the microstructure of open-cell foams and its effect on elastic properties. Int'l J. Solids \& Structures45, 1845-1875.

Jang, W.-Y., Kyriakides, S., 2009a. On the crushing of aluminum open-cell foams: Part I experiments. Int'l J. Solids \& Structures46, 617-634.

Jang, W.-Y., Kyriakides, S., 2009b. On the crushing of aluminum open-cell foams: Part II analysis. Int'l J. Solids \& Structures46, 635-650.

Jang, W.-Y., Kyriakides, S. and Kraynik, A.M. 2010. On the compressive strength of open-cell foams with Kelvin and random cell structures. Int'l J. Solids \& Structures47, 2872-2883.

Karagiozova, D., Langdon, G.S., Nurick, G.N. 2012. Propagation of compaction waves in metal foams exhibiting strain hardening. Int'l J. Solids \& Structures49, 2763-2777. 
Karagiozova, D., Alves, M., 2014. Compaction of a double-layered metal foam block impacting a rigid wall. Int'l J. Solids \& Structures51, 2424-2438.

Klintworth, J.W. and Stronge, W.J., 1988. Elasto-plastic yield limits and deformation laws for transversely crushed honeycombs. Int'l J. Mech. Sciences30, 273-292.

Knowles, J.K., 2002. Impact induced tensile wave in a rubberlike material. SIAM J. Appl. Math.62:4, 1153-1175.

Kraynik, A.M., Reinelt, D.A., van Swol, F., 2003. Structure of random monodisperse foam. Physical Review E67, 031403/1-11.

Kraynik, A.M., Reinelt, D.A., van Swol, F., 2004. Structure of random foam. Phys. Rev. Lett.93/20, 208301/1-4.

Papka, S.D. and Kyriakides, S., 1994. In-plane compressive response and crushing of honeycomb. J. Mechanics \& Physics of Solids42:10, 1499-1532.

Papka, S.D., Kyriakides, S., 1998a. In-plane crushing of a polycarbonate honeycomb. Int'l J. Solids \& Structures 35, 239-267.

Papka, S.D. and Kyriakides, S., 1998b. Experiments and full-scale numerical simulations of inplane crushing of a honeycomb. Acta Materialia46:8, 2765-2776, 1998.

Pattofatto, Elnasri, I., S., Zhao, H., Tsitsiris, H., Hild, F. and Girard, Y., 2007. Shock enhancement of cellular structures under impact loading: Part II analysis. J. Mech. Physics Solids $\mathbf{5 5}, 2672-2686$.

Reid, S.R. and Peng, C., 1997. Dynamic uniaxial crushing of wood. Int'l J. Imp. Eng.19, 531570 .

Ruan, D., Lu, G., Wang, B. and Yu, T.X. 2003. In-plane dynamic crushing of honeycombs - a finite element study. Int'l J. Impact Engin.28, 161-182.

Skews, B.W., Atkinks, M.D., and Seitz, M.W., 1991. Gas dynamic and physical behaviour of compressible porous foams stuck by a weak shock. Shock Waves, Proc. $18^{\text {th }}$ Int'l Symp. on Shock Waves, Sendai, Japan, Ed. K. Takayama, Vol. 1, pp. 511-516.

Tan, P.J., Reid, S.R., Harrigan, J.J., Zou, Z. and Li, S., 2005a. Dynamic compressive strength properties of aluminium foams. Part I - experimental data and observations. J. Mech. Phys. Solids53, 2174-2205. Tan, P.J., Reid, S.R., Harrigan, J.J., Zou, Z. and Li, S., 2005b. Dynamic compressive strength properties of aluminium foams. Part II - 'shock' theory and comparison with experimental data and numerical observations. J. Mech. Phys. Solids 53, 2206-2230. 
Zaretsky, E., Ben-Dor, G., 1995. Compressive stress-strain relations and shock Hugoniot curves for flexible foams. AMSE J. Eng. Mater. Technol.117, 278-284.

Zaretsky, E., Asaf, Z., Ran, E., Aizik, F. 2012. Impact response of high density flexible polyurethane foam. Int'l J. Impact Eng.39, 1-7.

Zheng, Z., Wang, C., Yu, J., Reid, S.R., Harrigan, J.J., 2014. Dynamic stress-strain states for metal foams using a 3D cellular model. J. Mech. Phys. Solids72, 93-114.

Zou, Z., Reid, S.R., Tan, P.J., Li, S., Harrigan, J.J. 2009. Dynamic crushing of honeycombs and features of shock fronts. Int'l J. Impact Eng.36, 165-176.

\section{Figure Titles}

Fig. 1 (a) A 3-D rendering of a random soap froth with $10 \times 10 \times 12$ cells. (b) Cross-sectional slice with ligaments dressed with beam elements.

Fig. 2 Quasi-static compressive response for foams of four different densities.

Fig. 3 Selected deformed configurations corresponding to points marked on the responses in Fig. 2.

Fig. 4 (a) Calculated initiation stresses, plateau stresses and densification strains and (b) crushing energy density both vs. relative density corresponding to the quasi-static crushing calculations.

Fig. 5 Slice of foam model crushed by shock; shows shock position and problem variables.

Fig. 6 (a) Proximal and (b) distal stress-time histories from direct impact simulationfor foams of four densities (constant impact speed $V^{+}=90 \mathrm{~m} / \mathrm{s}$ ).

Fig. 7 Selected deformed configurationscorresponding to the marked points on the responsesin Figs. 6a.

Fig. 8 Calculated shock speed-impact velocity Hugoniot for foams of four densities.

Fig. 9 Hugoniot strain vs. impact velocity for foams of four densities.

Fig. 10 Proximal $\left(\sigma^{-}\right)$and distal $\left(\sigma^{+}\right)$stresses vs. impact velocity for foams of four densities.

Fig. 11 Stresses vs. Hugoniot strain and the Rayleigh lines for $V^{+}=90 \mathrm{~m} / \mathrm{s}$ for foams of four densities.

Fig. 12 Energy density expended across shock vs. impact velocity for foams of four densities. 
690

691

692

693

694

695

Fig. 13 Proximal stress-displacement responses for quasi-static to supercritical impact velocities for foam models with (a) $3.67 \%$ and (b) $10.0 \%$ relative densities.

Fig. 14Deformed configurations of foam models impacted at different speeds. Images correspond to $\delta / h_{o}=0.15$ (see responses in Fig. 13). 